\title{
Behavioral risk factors of constipation in palliative care patients
}

\author{
Tomasz Dzierżanowski • Aleksandra Ciałkowska-Rysz
}

Received: 20 January 2014 / Accepted: 24 October 2014 / Published online: 4 December 2014

(C) The Author(s) 2014. This article is published with open access at Springerlink.com

\begin{abstract}
Background Constipation is frequently encountered in palliative care patients and remains a significant therapeutic problem. The etiology of constipation is multifactorial. Nutritional and behavioral factors are considered common causes of constipation; however, their impact has not yet been assessed precisely.

Objective The aim of this study was to assess the correlation between the frequency of bowel movements (FoBM) and risk factors of constipation in palliative care patients.

Design and subjects A cohort retrospective study was performed in three palliative care centers, including outpatient, home, and inpatient care cancer patients using questionnaires on bowel dysfunction symptoms, behavioral risk factors, and opioid use. The inclusion criterion was adult patients examined on the day of admission. The exclusion criterion was Karnofsky performance status score $\leq 20$.

Measurements Spearman's rank correlation coefficient was used to measure the statistical dependence between two variables and frequency analysis was performed using the chisquared test and Fisher's exact test.

Results Two hundred thirty-seven valid questionnaires were collected. We found the correlation between FoBM and insufficient food and fluid intake $(p<0.0001)$, as well as for inadequate conditions of privacy ( $p=$ $0.0008)$, dependency on a caregiver $(p=0.0059)$, and the patient's overall performance status $(p=0.013)$. We did not manage to prove bed rest as the independent risk factor of constipation.
\end{abstract}

T. Dzierżanowski $\cdot$ A. Ciałkowska-Rysz

Palliative Care Unit, Medical University in Lodz, Lodz, Poland

T. Dzierżanowski $(\square)$

Gastroenterology Clinic CSK MSW, Warsaw, Poland

e-mail: tomasz.dzierzanowski@umed.lodz.pl
Conclusions The main risk factors of constipation in palliative care patients appeared to be insufficient fluid and food intake, inadequate conditions of privacy, dependency on a caregiver, as well as poor general performance status.

Keywords Constipation $\cdot$ Palliative care $\cdot$ Prevention

\section{Background}

The Polish Society of Palliative Medicine (PTMP) worked out the recommendations to prevent and manage constipation in palliative care patients (PTMP Guidelines 2009) [1]. This document defines constipation as decreased frequency of bowel movements (less than 3 per week), or subjective symptoms, such as difficulty in defecation, hard stools, straining, and feeling of incomplete bowel evacuation. It is one of the most common symptoms observed in palliative care patients $[2,3]$. There may be several concomitant causes of constipation in these patients $[4,5]$. Emphasis is placed on behavioral causes, such as those related to immobilization, disability, deconditioning, and insufficient fluid and food intake. These behavioral risk factors seem to be essential in the etiology of occurrence and degree of constipation. The importance of particular risk factors is not yet assessed; however, they have been considered for the development of a constipation risk assessment scales [6]. The current clinical guidelines, including those of the Polish Society of Palliative Medicine in 2009, include counteracting risk factors. However, these recommendations are based on the expert opinion, due to scarce clinical data.

The PTMP recommends monitoring of constipation symptoms and determining the risk factors using the available tools and scales such as the Bowel Function Index; however, it appeared difficult to implement them as the existing formal documentation and procedures 
were insufficient for this purpose. The formal questionnaires required by the Ministry of Health were too general and useless for precisely diagnosing and monitor constipation. The PTMP supported a pilot test with a use of a new questionnaire (the Bowel Function Assessment Questionnaire, BFAQ; Table 1) developed by the author of this paper. The aim of that pilot implementation was to find the feasible and precise diagnostic tool that would improve the existing method [7]. The questionnaires were filled out by nurse or physician. The BFAQ was implemented as the pilot tool in three palliative care centers, aimed to standardize mandatory routine diagnostics of constipation as well as to monitor of the effectiveness of prevention and treatment, as was recommended by the PTMP [1].

Table 1 Studied population

\begin{tabular}{|c|c|c|}
\hline & Number of samples & Percentage \\
\hline Population & 237 & \\
\hline Men & 126 & 53 \\
\hline Women & 111 & 47 \\
\hline \multicolumn{3}{|l|}{ Age } \\
\hline Mean & 68 & \\
\hline Minimum & 26 & \\
\hline Maximum & 92 & \\
\hline $\mathrm{SD}$ & 12 & \\
\hline \multicolumn{3}{|l|}{ Form of care } \\
\hline Inpatient & 137 & 58 \\
\hline Home & 41 & 17 \\
\hline Outpatient clinic & 57 & 24 \\
\hline N/A & 2 & 1 \\
\hline \multicolumn{3}{|l|}{ Primary cancer site } \\
\hline Women & 111 & \\
\hline Lung & 23 & 20.7 \\
\hline Breast & 21 & 18.9 \\
\hline Female sex organs & 13 & 11.7 \\
\hline Colorectal & 7 & 6.3 \\
\hline Pancreas & 4 & 3.6 \\
\hline Other neoplasms & 37 & 33.3 \\
\hline N/A & 6 & 5.4 \\
\hline Men & 126 & \\
\hline Lung & 45 & 36.6 \\
\hline Prostate & 20 & 16.3 \\
\hline Colorectal & 8 & 6.5 \\
\hline Urinary bladder & 8 & 6.5 \\
\hline Kidney & 7 & 5.7 \\
\hline Other & 35 & 28.5 \\
\hline N/A & 3 & 2.4 \\
\hline Opioid use & 181 & 76.4 \\
\hline Laxative use & 173 & 73.0 \\
\hline
\end{tabular}

\section{Aim}

The aims of the study were to test the following:

1. In palliative care patients, is there a correlation between the frequency of bowel movements and the following behavioral factors?

- Immobilization (bed rest)

- Insufficient fluid intake

- Insufficient food intake

- Inadequate privacy during defecation

- Decreased activity of daily life

2. In palliative care patients, is there a correlation between the frequency of bowel movements and the general performance status (the Karnofsky score)?

\section{Methods}

A retrospective cohort study was conducted in three hospice centers including all forms of palliative care: home care, outpatient clinic, palliative ward, and inpatient hospices. We analyzed the data collected by qualified nurses and physicians using the Bowel Function Assessment Questionnaires. One questionnaire per patient was filled out by a physician or nurse as a routine diagnostic tool for all patients on the day of admission to the appropriate inpatient or outpatient care unit during the observational period, excluding patients with very poor overall performance status (Karnofsky 10-20). The reliability and feasibility of the BFAQ tool was tested [7].

Only properly filled-out questionnaires were taken into the analysis. Illegible or inaccurately filled-out questionnaires were rejected. No other exclusion criteria were set to ensure the involvement of a wide range of patients.

The frequency of bowel movements was defined as the total number of days during the last 7 days with at least one effective defecation. Bed rest was measured as the average percentage of a day spent in bed during the last 7 days. This information was collected by a nurse/physician from the patient or his or her caregivers.

Difficulty with bowel movements was the patient's subjective assessment of ease of defecation during the last 7 days on an 11-point numeric scale ( 0 - no difficulty; 10 - severe difficulty).

Insufficient fluid intake and insufficient nutrition were assessed using the 5-grade Likert scale: 0 -no insufficiency; 1 -insignificant nutrition/fluid intake impairment (some attention was required to provide adequate nutrition/fluid intake); 2-moderate nutrition/fluid intake impairment; 3-major nutrition/fluid intake impairment (fluids/food taken with 
effort); 4 - significant insufficiency, during the last 7 days. This was based on a patient and his caregivers' assessment, and not a calculation of real caloric intake.

For privacy conditions during bowel movements, the Likert scale was used as well: 0 - privacy ensured; 1 - privacy ensured, but others' assistance was required after defecation; 2 - privacy was impaired by the presence of other patients or persons in the room during defecation; 3-privacy was impaired by the need of assistance during defecation; 4-no privacy ensured. The nurse/physician rated privacy based on the observation of the environment conditions as well (e.g., an open door during defecation, presence of other persons). The real patient's perception might differ from the assessment of the nurse/physician.

The questions, as well as the Karnofsky performance status (KPS), were rated by a physician or nurse. The detailed instruction was amended to the BFAQ to ensure standardized assessment.

Other data on opioid and laxative use were collected for separate complex analysis of iatrogenic causes.

The study received approval by an ethical committee.

\section{Statistical analysis}

The Kruskal-Wallis one-way analysis of variance was applied for nonparametric data.

Spearman's rank correlation coefficient was used to measure statistical dependence between two variables. A $p$ values less than 0.05 were considered statistically significant, and the Bonferroni correction was applied for multiple comparisons.

Frequency analysis was performed using the chi-squared test and Fisher's exact test.

The data was analyzed by Statistica 10 (StatSoft).

\section{Results}

Structure of studied population

Of the 273 cancer patients screened, 237 properly filled out questionnaires were qualified for the analysis. Sixty percent of the questionnaires were collected and scored by nurses and $40 \%$ by physicians. Thirty-six (13\%) questionnaires were discarded as they were illegible or neglectfully filled out.

The population consisted of 111 women and 126 men (47 and $53 \%$, respectively), aged 26 to 92 years (mean 68 years; SD 12.2 years). The demographic details are depicted in Table 2.

The majority ( $58 \%$ ) were inpatient hospice cancer patients (except one patient with respiratory failure). The most frequent primary sites of neoplasm were lung $(21 \%)$ and breast $(19 \%)$ in women and lung (36\%) and prostate $(16 \%)$ in men.
Table 2 The elements of the bowel function assessment questionnaire (all questions concern the last 7 days)

1.Demographic data: age, gender, basic diagnosis ICD-10, probable weight, and form and place of palliative care

2.Objective measures of bowel movements

a.The last bowel movements [days]

b.Number of bowel movements [days]

3.Bowel Function Index [NRS 0-10]

a.Ease of defecation during the last seven days according to the patient's judgment

$(0=$ easy/no difficulty; $10=$ severe difficulty).

b.Feeling of incomplete bowel evacuation during the last 7 days according to patient's assessment $(0=$ not at all; $10=$ very strong $)$ c.Personal judgment of patient regarding constipation $(0=$ not at all; $10=$ very strong)

4.The Patient Assessment of Constipation Symptoms (PAC-SYM) questionnaire [Likert $0-4]$

5.Risk factors

a.Bed rest [\% of day activity]

b.Insufficient fluid intake [Likert 0-4]

c.Insufficient nutrition [Likert 0-4]

d.Insufficient privacy during bowel movements [Likert 0-4]

e.Dependence on the carer [Likert 0-4]

6.Karnofsky performance status scale [\%]

7.Mean daily opioid burden [mg] as equianalgesic morphine dose

8.Constipation prophylaxis and treatment (dietary, oral laxatives,

suppositories, enema, methylnaltrexone s.c., manual stool evacuation)

9.Other drugs

The correlation between the frequency of bowel movements and behavioral risk factors

\section{Bed rest}

The degree of immobilization was measured as the percentage of daily activity spent in bed and its mean was $62 \%$, with the full range of the values possible (0-100\%); however, there were significant differences between inpatient, outpatient, and home care patients (Fig. 1a; $p<0.001$ ). Home care patients appeared much more immobilized than inpatient and ambulatory care patients (mean 44.7 vs. 19.5 and $8.5 \%$, respectively). Spearman's rank coefficient of correlation between frequency of bowel movements and the degree of bed rest was -0.13 ( $p=0.046)$; however, with the Bonferroni correction, it appeared insignificant.

\section{Insufficient fluid intake and nutrition}

Insufficient fluid intake and nutrition were assessed using the Likert scale. The mean for fluid and nutrition intake came to 1.4 on the Likert 0-4 scale (SD 1.1 and 1.2, respectively; values ranging from 0 and 4). Spearman's rank coefficients of correlation between bowel movements and proper fluid/ nutrition 
intake were -0.26 and -0.32 , respectively $(p<0.0001)$. Figure $1 \mathrm{~b}, \mathrm{c}$ depicts the distribution of the assessments in the particular forms of palliative care. No statistical differences of distribution were found between these subpopulations ( $p=$ 0.0387 for fluid intake and $p=0.1148$ for nutrition).

\section{Inadequate privacy during bowel movements}

Privacy during defecation was also assessed using the Likert 0 4 scale too (mean 1.0; SD 1.3). There was a statistical difference observed between outpatient and inpatient care patients observed $(p<0.0001)$, but none with the home care population (Fig. 1d). Spearman's rank coefficient of correlation between bowel movements and privacy was $-0.22(p=0.0008)$.

\section{Dependence on the caregiver}

The dependence on the caregiver was assessed in the Likert 04 scale, with mean of 1.9 (SD 1.6), and there were significantly lower values in the outpatient population than in the home and inpatient groups (Fig. 1e).

Spearman's rank coefficient of correlation between bowel movements and the dependence on the caregiver came to -0.18 and was statistically significant $(p=0.0059)$.

\section{The relationship between the frequency of bowel movements and the Karnofsky performance status score}

The mean Karnofsky performance status score was 51.2 (range 20-90; SD 17.6) and was significantly higher in outpatient patients $(p<0.0001$; Fig. 1f).

Spearman's rank coefficient of correlation between bowel movements and the Karnofsky score was $0.16(p=0.013)$.

\section{Discussion}

Constipation affects $42.4 \%$ of end-stage oncology patients and is one of the most frequent symptoms besides pain and cachexia [8]. In 85-95\% of cases, constipation results in decreased health-related quality of life, although two thirds are of mild and moderate intensity [9]. They also generate additional financial burden for the health system [10]. This study was aimed to assess the impact of behavioral factors on the frequency of constipation, especially those that a nursing staff may have an influence on and be able to modify to decrease the risk of constipation in their patients. The expert taskforce of the Polish Society of Palliative Medicine recommended using symptom assessment scales in diagnosing and monitoring constipation [1]. The implementation of the Bowel Function Assessment Questionnaire (BFAQ) was a pilot implementation of such guidelines used in three palliative care centers to verify the feasibility and usefulness of such a tool. It allowed to collect data in a systematic way on the intensity of symptoms in constipation, its risk factors, as well as to analyze the correlation between the assessment tools. This study's main focus was on the correlation of behavioral factors, as well as nursing risk factors, with constipation.

There is no commonly agreed definition of constipation. It typically consists of both objective and subjective symptoms [11-13]. One of the objective symptoms that is easy to assess is frequency of bowel movements, but one which is insensitive to patient's subjective experiences; therefore, we assume that the frequency should not be the only predictor of constipation. In palliative care patients, the subjective symptoms, such as straining, incomplete defecation, excessively and hard stools, are even more important, as they decide on the impairment of the health-related quality of life [14]. In this study, we used the officially recommended PTMP definition of constipation with the frequency of bowel movements less than 3 per week or difficulty of defecation of more than 4 in $0-11$ numerical rating scale. However, in another analysis, we not only demonstrated the high correlation between the frequency of bowel movements and patient's subjective symptoms, but also found that the frequency of less than 4 bowel movements results in significant suffering of patients. In our opinion, diagnosing constipation based on the frequency of less than 3 bowel movements per week may already lead to unnecessary suffering [12].

The studied population included adult patients with a wide age range (26 to 92 years) with a slight predominance of men $(53 \%)$. Patients from all three forms of palliative care available in Poland were included: inpatient hospice, home care, and outpatient (ambulatory) clinics. Most of them were inpatient hospice patients ( $58 \%$ ). The distribution of the primary site of neoplasm is typical for Polish population (see Table 2).

The most important risk factors appeared to be insufficient fluid and food intake, which is congruent with common observation on this topic. It is worth underlining that patients both in inpatient and home care are affected by these risk factors to a similar extent, regardless of whether they were inpatient or home care. Thus, ensuring proper supply of fluids and food is crucial for bowel functioning and forming stool. Dietary recommendations should be communicated thoroughly to caregivers at home, as should be to those providing care in inpatient hospices. However, we understand that proper supply of fluids and food is in many cases difficult or even impossible to modify, due to the patient's condition.

Defecation is an intimate act, so when privacy is not ensured, patients may refrain from bowel movements that leads to habitual constipation. Ensuring privacy during bowel movements is a simple, cost-effective intervention. Educating the nursing staff to treat a patient with respect to his/her dignity honors a patient's basic right to privacy. In this study, 


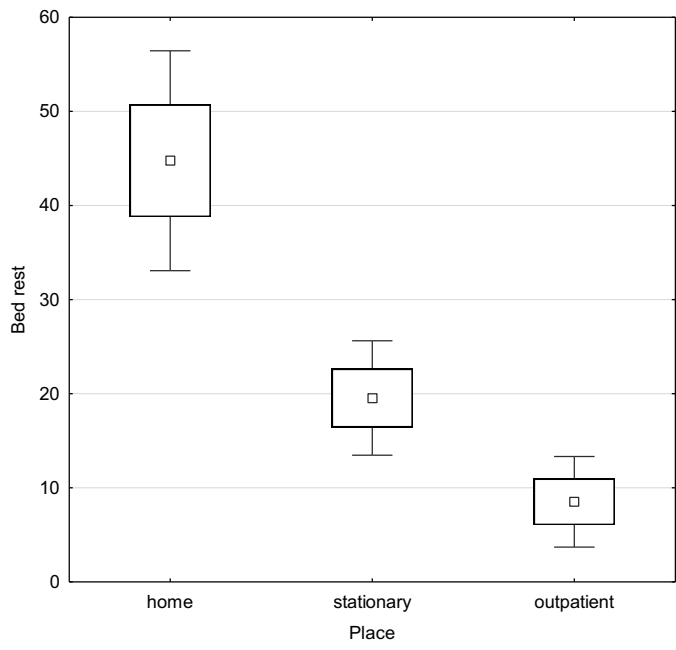

A.
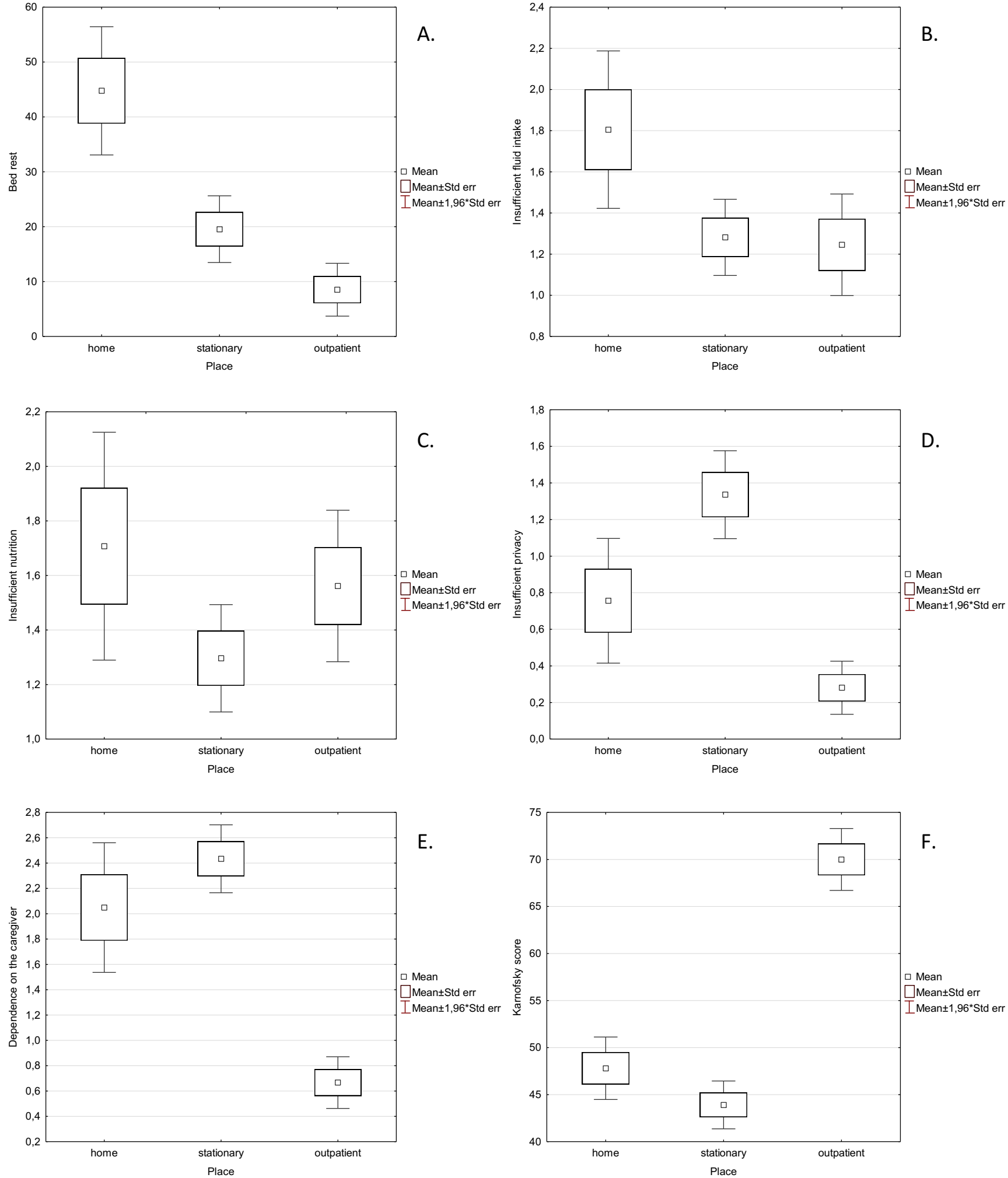

Fig. 1 The correlation between the frequency of bowel movements and behavioral factors and Karnofsky performance status score

we revealed that this factor has significant impact on the patient's ability to defecate. The least impaired patients that are able to be managed in outpatient clinics usually are also able to take care of themselves. Thus, privacy is easier to attain in this group than in inpatient hospice patients. In the latter group, patients usually defecate assisted by the nursing staff. In many cases, it is possible to arrange the care and a patient's room in such way that ensures privacy. 
Many patients lack the ability to use a toilet on their own. In such cases, the caregiver assists a patient during defecation. The accessibility of a caregiver on demand appears necessary, as patients may refrain from bowel movements until a caregiver comes. Postponing defecation is one cause of habitual constipation.

Immobilization and low physical activity are commonly regarded as real factors for constipation. Based on the data collected, we did not find statistical significance for bed rest as a risk factor for constipation, when applying the Bonferroni correction for multiple comparisons $(p=0.046$ exceeded the accepted value 0.008 ). Though this result needs to be taken with caution. Not only are bedridden patients prone to being immobilized, but also patients in wheelchairs and all those that do not perform any exercises. Further investigations with more precise assessment are required. We have also found that home care patients were surprisingly much more bedbound than inpatient ones, which does not fit with our common observation. On the other hand, defecation is more troublesome and difficult when a patient remains supine, so habitual constipation may be more frequent in bedridden patients. The PTMP recommends simple nursing procedures such as providing unexhausting physical activity for the patient, providing and respecting privacy during defecation. The dependence on a caregiver may also be diminished by using supports such as WC-chairs and safety rollers [1].

The performance status score appeared to be correlated with the frequency of bowel movements $(p=0.013$ exceeded the Bonferroni-corrected $p$ value; however, we support that it should be accepted anyway). We suggest focusing on this factor for screening purposes and in all patients where the assessment of risk factors is impossible. The lower the performance status score, the higher likelihood of constipation. Thus, one may anticipate constipation in patients with deterioration of a patient's status. It is obvious that as their physical activity diminishes, patients spend more time in bed, subsequently losing appetite and thirst. Along with progression of disease, they become dependent on their caregivers to a higher degree and are prone to situations uncomfortable for defecation. So along with deterioration of general performance status, the problem of constipation arises and intensifies.

In several epidemiologic surveys, age is indicated as a risk factor of constipation. In people older than 75 years, constipation is twice as frequent as in those under 75 years of age. Similarly, in demented patients, it is twofold more frequent [15]. It needs to be kept in mind though that it can be related to factors such as lower physical activity, lower thirst, and appetite. Also, in our study, we found that bed rest increases with age $(p=0.0002)$. We did not find age as a statistically significant factor of constipation though. However, palliative care patients differ from people in the general population, as they are affected by many factors usually not present in healthy persons.
There are other treatment-derived causes of constipation in palliative care patients. The most important are opioid analgesics which produce opioid bowel dysfunction syndrome (OBD). It is the most frequent problem in opioid treatment and affects $70-90 \%$ of these patients [16]. There are also many possible other concomitant causes of constipation in these patients. The Polish Society of Palliative Medicine (PTMP) defined the opioid induced constipation as constipation in which the possible cause is opioid use, given that it is impossible to indicate which other factors are responsible for bowel dysfunction in the same time. Opioid-induced constipation is the toughest problem during pain treatment using opioid analgesics. It is usually poorly responsive to regular laxatives and may be an obstacle to effective pain management [17]. In addition, unlike somnolence, nausea, and vomiting, constipation does not decrease but increases with the length of opioid treatment [18]. The expert taskforce of PTMP strongly recommended to consider all other possible causes of constipation even though opioids might appear the obvious one [1]. We excluded opioid treatment from this study for a separate analysis; however, the incidence of constipation was significantly higher in patients taking opioids despite laxative use. Laxatives were used in the majority (73\%) of all the patients and more frequently in those treated with opioids.

Dietary recommendations may often be difficult to follow. For example, a cachectic patient may be unable to take in sufficient amount of food and fluids to ensure effective gut transit and proper consistency of feces. Fiber supply and other volume increasing supplements may even be contraindicated in such cases. However, frequent small amounts of food and water is regarded as effective prevention against constipation. Further investigation is required though.

The findings of this study give some hints for clinical practice. First, they support the PTMP guidelines to counteract all the reversible causes of constipation. Second, they reflect the outstanding importance of common risk factors that may be in many cases addressed to every day care, some of them do not require additional spending, but only an increase of the awareness and routine attention. However, it may be sometimes difficult to achieve sufficient fluid intake or to mobilize a patient adequately, these key aspects should be firstly addressed and should forestall pharmacotherapy.

Most patients with cancer pain receive at least satisfactory relief [19]. Constipation seems to be one of the unsolved causes of suffering in palliative care patients, and once it develops, it is very difficult to treat. Thus the prevention, including management of risk factors, remains pivotal in the treatment strategy.

Conflict of interest This research received no specific grant from any funding agency, public or commercial, or from nonprofit sectors. 
Open Access This article is distributed under the terms of the Creative Commons Attribution Noncommercial License which permits any noncommercial use, distribution, and reproduction in any medium, provided the original author(s) and the source are credited.

\section{References}

1. Leppert W, Dzierżanowski T, Ciałkowska-Rysz A, Jarosz J, Pyszkowska J, Stachowiak A. The management of constipation in palliative medicine - recommendations of the Expert Working Group of the Polish Association for Palliative Medicine. Medycyna Paliatywna 2009; 1:1:10

2. Sykes NP (1998) The relationship between opioid use and laxative use in terminally ill cancer patients. Palliat Med 12: 375-382

3. Potter J, Hami F, Bryan T, Quigley C (2003) Symptoms in 400 patients referred to palliative care services: prevalence and patterns. Palliative Medicine 17:310-314

4. Hsieh C (2005) Treatment of Constipation in Older Adults. Am Fam Physician 72:2277-2285

5. Dzierżanowski T (2012) Opioid induced bowel dysfunction in cancer patients. Medycyna Paliatywna 2:57-66

6. Richmond JP, Wright ME (2006) Development of a constipation risk assessment scale. Journal of Orthopaedic Nursing 10:186-197

7. Dzierżanowski T, Ciałkowska-Rysz A, Jarosz J (2010) The assessment of the diagnostic tools for constipation in palliative care patients. Medycyna Paliatywna 2:81-91

8. Clark K, Smith JM, Currow DC (2012) The prevalence of bowel problems reported in a palliative care population. J Pain Symptom Manage 43(6):993-1000

9. Bell T, Milanova T, Grove G, Williamson R, Bolge S. OBD symptoms impair quality of life and daily activities, regardless of frequency and duration of opioid treatment: results of a US patient survey (PROBE survey). The Journal of Pain 2007; 8, :S71
10. Annunziata K, Bell T (2006) Impact of opioid-induced constipation on healthcare resource utilisation and patient functioning. European Journal of Pain 10:S172

11. Lembo A, Camilleri M (2003) Chronic constipation. N Engl J Med 349(14):1360-8

12. Dzierżanowski T, Ciałkowska-Rysz A (2011) Assessment of the usefulness of the definition of constipation in palliative care patients according to Polish Society of Palliative Medicine. Medycyna Paliatywna 1:33-38

13. Longstreth GF, Thompson WG, Chey WD, Houghton LA, Mearin F, Spiller RC (2006) Functional bowel disorders. Gastroenterology 130(5):1480-91

14. Slappendel R, Simpson K, Dubois D, Keininger DL (2006) Validation of the PAC-SYM questionnaire for opioid-induced constipation in patients with chronic low back pain. European Journal of Pain 10:209-217

15. Talley NJ, Jones M, Nuyts G, Dubois D (2003) Risk Factors for chronic constipation based on a general practice sample. Am J Gastroenterol 98(5):1107-11

16. Lucero M, Von Scheele B, Blackard R, Milanova T, Bell T (2006) The incidence and impact of gastrointestinal adverse events (GIAEs) associated with opioid analgesic use: a review of the literature. The Journal of Pain 7:S89

17. Mercadante S, Villari P, Ferrera P, Casuccio P (2006) Opioid-induced or pain relief-reduced symptoms in advanced cancer patients? European Journal of Pain 10:153-159

18. Annunziata $\mathrm{K}$ et al. Constipation is a predominant side effect of opioid treatment for persistent pain. The Journal of Pain, Volume 2006; 7:S89

19. Zech DF, Grond S, Lynch J, Hertel D, Lehmann KA (1995) Validation of World Health Organization Guidelines for cancer pain relief: a 10-year prospective study. Pain 63:65-76

The questionnaire and the example data are available on demand - please contact the authors directly: tomasz.dzierzanowski@umed.lodz.pl. 